Bundesgesundheitsbl 2019 · 62:580-588 https://doi.org/10.1007/s00103-019-02941-x Online publiziert: 17. April 2019

(c) Der/die Autor(en) 2019

Hendrik Siebert ${ }^{1} \cdot$ Helmut Uphoff ${ }^{2} \cdot$ Henny Annette Grewe ${ }^{1}$

'Public Health Zentrum, Hochschule Fulda, Fulda, Deutschland

${ }^{2}$ Fachbereich Infektionsepidemiologie, Hessisches Landesprüfungs- und Untersuchungsamt im Gesundheitswesen, Dillenburg, Deutschland

\title{
Monitoring hitzebedingter Sterblichkeit in Hessen
}

ren folgen dabei immer demselben Prinzip, nämlich der Berechnung der Abweichung eines Istwertes der täglich auftretenden Ereignisse während Hitzeperioden von einem Wert, der die erwartete Sterblichkeit ohne Hitzeeinfluss darstellt. In der Datenauswahl, ihrer Erfassung, im Datentransfer, in den Auswertungsmethoden, den Beurteilungskriterien und den Kommunikationspfaden unterscheiden sich die etablierten Systeme allerdings erheblich. Überwiegend handelt es sich dabei um mortalitätsbasierte Systeme. Sie sind von nationalen Besonderheiten geprägt, die von unterschiedlichen Definitionen eines Hitzeereignisses bis zu unterschiedlichen Kriterien für eine Übersterblichkeit reichen. So verwendet das belgische System zur Definition eines Hitzeereignisses eine fixe Schwelle von $25^{\circ} \mathrm{C}$ Maximaltemperatur in Kombination mit einem Zeitkriterium [10]. Andere Systeme operieren mit relativen Temperaturkriterien unter Verwendung perzentilbasierter Ansätze [11, 12] oder eigens entwickelter Indikatoren, die verschiedene Kriterien zu einem Index zusammenfassen [13]. Übersterblichkeit (Exzessmortalität) wird in allen Systemen gleichfalls durch Schwellenwertsetzung definiert. Exzesssterbefälle ergeben sich, wenn die beobachtete Anzahl an Sterbefällen diese Schwelle überschreitet. Dabei unterscheiden sich die statistische Definition der Schwellenwerte (Standardabweichung, z-Scores, Konfidenzintervalle) sowie die Verfahren zu deren Schätzung. In der Regel werden Referenzperioden ausgewählt, auf deren Grundlage Erwartungswerte zur Konstruktion einer Basislinie geschätzt werden, die in ihrem Verlauf das charakteristische Sterbege- schehen in den Sommermonaten abbildet $[10,11,13-15]$. Die Addition von Basislinie und Schwellenwert bildet dann das statistische Exzesskriterium, ab dem ein Sterbefall als Exzesssterbefall angesehen wird. Den Verfahren gemein ist die Verwendung des Sterbegeschehens unabhängig von Todesursachen (Gesamtmortalität).

Das Monitoring hitze(mit)bedingter Todesfälle in Baden-Württemberg unterscheidet sich in Zielsetzung und Methodik deutlich von den o.g. Verfahren. Ziel ist die Erfassung stattgehabter Häufungen von Todesfällen während der Sommermonate Juni bis August auf der Basis monatlich aggregierter Daten unabhängig von nachweislichen Temperaturextremen, insofern ohne Schwellenwertdefinition für „Hitze“ [16]. Die Erwartungswertschätzung der monatlichen Sterblichkeit erfolgt durch Auswahl derjenigen Sommermonate der Jahre 1970 bis 2015, die, gemessen an einem Referenzzeitraum (April bis November), den geringsten Anteilswert aller in diesem Referenzzeitraum aufgetretenen Sterbefälle zeigten. So wurde zur statistischen Schätzung von hitzebedingten Exzesstodesfällen im Monat Juni der Anteilswert des Junis aus dem Jahre 1992 herangezogen. Daraus wird der Erwartungswert der monatlichen Anzahl von Sterbefällen berechnet. Die Differenz zwischen den beobachteten und erwarteten Fallzahlen wird schließlich als hitzebedingter Exzess definiert. Auf diese Weise wurden für die Jahre 2000 bis 2017 insgesamt 24.306 hitzebedingte zusätzliche Sterbefälle während der Sommermonate geschätzt. Über dieses Verfahren sollen 


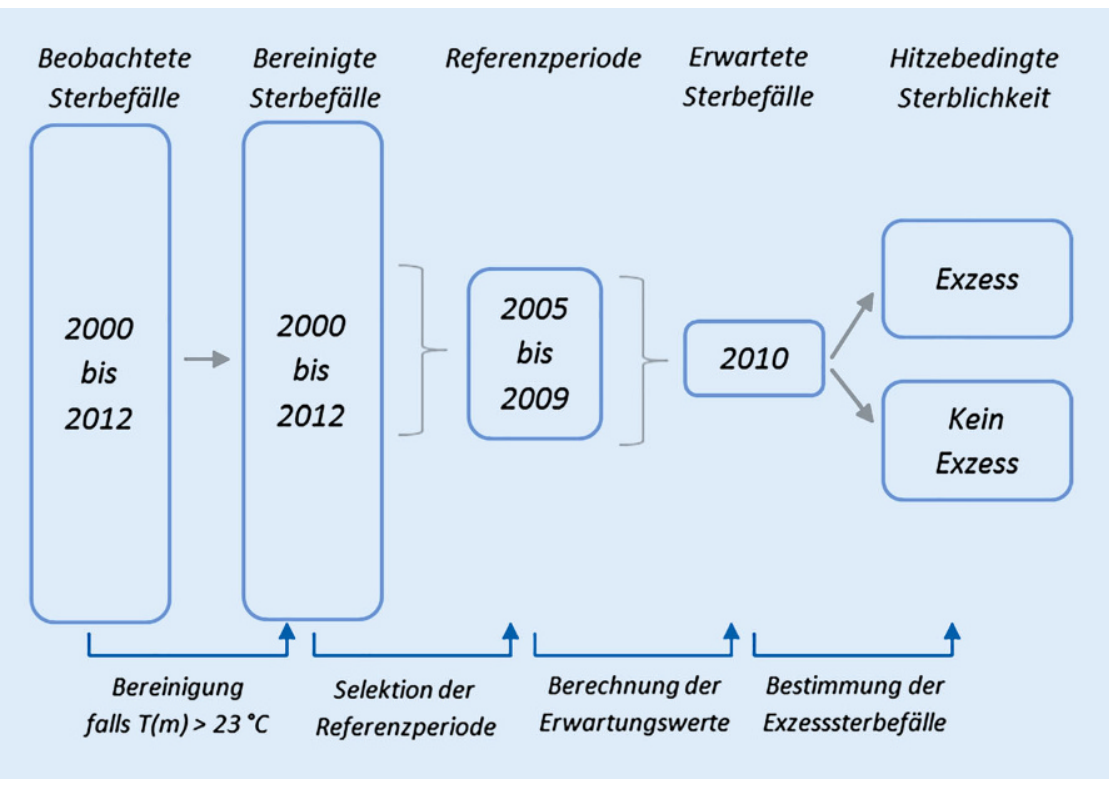

Abb. 1 A Algorithmus zur Schätzung der hitzebedingten Exzessmortalität im Bundesland Hessen. Beispiel für das Auswertungsjahr 2010 mit Referenzperiode 2005-2009 (Projekt HEAT II). T(m) mittlere Tagesmitteltemperatur, gemittelt über alle datenliefernden Wetterstationen

Verzerrungen durch demografische Veränderungen minimiert werden [17].

Mit der Euro-MOMO(European monitoring of excess mortality for public health action)-Initiative (www. euromomo.eu) wurde seit 2009 der Versuch unternommen, die Sterblichkeit in europäischen Staaten nach einheitlichen Kriterien darzustellen. EuroMOMO beobachtet die Mortalität in bis zu 23 datenliefernden Ländern bzw. Regionen und stellt ganzjährig sowohl länderübergreifende als auch länderspezifische Indikatoren auf wöchentlicher Basis bereit, die als Ergebnis eines eigens entwickelten Auswertungsalgorithmus generiert werden [18]. Dieser schätzt die Erwartungswerte unter Rückgriff auf kleinere Zeitreihenausschnitte aus den Frühlings- und Herbstmonaten (Kalenderwochen 16-25 und 37-44) aus einer drei- bis maximal fünfährigen Referenzperiode durch ein Poisson-Modell. Hierfür wird angenommen, dass diese Ausschnitte nicht von externen, azyklischen Geschehen wie Influenza oder Extremwetterereignissen beeinflusst sind, das Sterbegeschehen folglich unverzerrt repräsentieren und daher für die Schätzung des Sterbegeschehens in den übrigen Abschnitten des Jahres geeignet sind. Darüber hinaus werden die Ergebnisse für die häufig verzögerte
Bereitstellung vollzähliger Sterbedaten aus den datenliefernden Ländern durch Prädiktion adjustiert. Euro-MOMO war und ist primär auf ein Monitoring der Influenzaaktivität ausgerichtet und integriert bislang keine länderspezifischen Temperaturschwellen für Hitzeereignisse.

Vor dem Hintergrund eines fehlenden "Goldstandards" zur Ermittlung hitzebedingter Exzesssterblichkeit und der Notwendigkeit, länderspezifische Besonderheiten in der Datenverfügbarkeit sowie den Ressourcen für die Datenauswertung zu berücksichtigen, stellte sich die Frage nach einem möglichst pragmatischen, aufwandsarmen, datensparsamen sowie für alle Akteursgruppen methodisch gut nachvollziehbaren Verfahren zur Schätzung hitzebedingter Übersterblichkeit.

\section{Methoden}

Es wurde der international etablierte Ansatz eines formalen Vergleichs der beobachteten und der für den gleichen Zeitraum erwarteten Anzahl an Sterbefällen verfolgt. Für die Entwicklung des Verfahrens standen zunächst die hessischen Sterbefallzahlen der Jahre 2000 bis 2012 zur Verfügung. Mithilfe dieser Datenreihe wurde ein Algorithmus sowohl für die Schätzung der hitzeassoziierten Ex- zesssterbefälle des Bundeslandes Hessen als auch für kleinräumige Auswertungen nach klimatischen Regionen entwickelt. Die Überprüfung des Verfahrens unter dem Aspekt seiner Praktikabilität im Routinebetrieb erfolgte sodann mit den hessischen Sterbezahlen der Jahre 2013 bis 2018 (bis August) für Hessen, sodass in Summe Auswertungsergebnisse von 2005 bis zum Sommer 2018 vorliegen.

Für die Ermittlung der Temperaturschwelle, ab der ein Sterbeexzess als "wahrscheinlich durch Hitze mitbedingt" eingeschätzt werden kann, wurde ein tägliches Flächenmittel als arithmetisches Mittel (mittlere Tagesmitteltemperatur) aus den Tagesmitteltemperaturen der hessischen Wetterstationen berechnet. Mit der Tagesmitteltemperatur wurde ein Parameter gewählt, der in der Lage ist, Tages- und Nachtwerte und damit z. B. tropische Nächte und ausbleibende nächtliche Abkühlungsphasen zu repräsentieren. In der Entwicklungsphase (2000 bis 2012) wurden die Temperaturdaten aus den Messnetzen des Deutschen Wetterdienstes (DWD) sowie des Hessischen Landesamtes für Naturschutz, Umwelt und Geologie (HLNUG) entnommen und als Flächenmittel aggregiert. Ein Vergleich der als Flächenmittel aggregierten Wetterdaten aus beiden zugänglichen Messnetzen mit den Flächenmitteln nur eines Messnetzes ergab keinen relevanten Unterschied, sodass unter Praktikabilitätsgesichtspunkten ein Datensatz, in den die Messungen flächendeckend verteilter Wetterstationen eingehen, als hinreichend angesehen wird. Für den Testlauf im Routinebetrieb (2013 bis 2018) wurden daher lediglich die Daten von 35 Wetterstationen des DWD nach dem geschilderten Verfahren aggregiert. Wie sich in Vergleichstests zeigte, reicht es für kleinräumigere Betrachtungen aus, die Daten repräsentativer einzelner Wetterstationen heranzuziehen.

Unter den Annahmen eines hinreichend kontinuierlichen, charakteristischen Jahresganges der Sterbefälle und einer nur allmählichen Auswirkung langfristiger Trends im demografischen Strukturwandel wurden die Erwartungswerte im Rahmen der Entwicklungspha- 
se aus den beobachteten Sterbefällen jeweils fünführiger Referenzperioden innerhalb der Jahre 2000 bis 2012 unter Verwendung des entwickelten Algorithmus geschätzt (• Abb. 1). Demnach ließen sich zunächst Erwartungswerte für acht Auswertungsjahre (2005 bis 2012) berechnen.

Die Erwartungswerte für jeden Kalendertag eines jeden Auswertungsjahres wurden mit einem ungewichteten, gleitenden Durchschnitt aus jeweils 25 Beobachtungswerten (fünf Kalendertage $\times$ fünf Jahre) der jeweiligen Referenzperiode berechnet (• Abb. 2).

Um den potenziell verzerrenden Einfluss von Übersterblichkeit aufgrund von Hitze innerhalb der Referenzperioden zu begrenzen, wurden die beobachteten täglichen Sterbefälle durch den Median der Fallzahlen drei aufeinanderfolgender Kalendertage aus jeweils drei vorherigen Jahren ersetzt, sofern die mittlere Tagesmitteltemperatur des $\mathrm{zu}$ ersetzenden Tages eine Schwelle von $23^{\circ} \mathrm{C}$ überschritten hatte. Die Tage mit bereinigten Fallzahlen (gelb markierte Tage in - Abb. 2) wurden anstelle der beobachteten Fallzahl für die Erwartungswertschätzung verwendet. Diese Expositionsschwelle wurde abgeleitet aus der Prüfung, wann die für kleine Temperaturintervalle (jeweils $1^{\circ} \mathrm{C}$ ) beobachtete relative Häufigkeit von Tagen mit Sterbeexzessen über einen Bereich von $7,5-27,5^{\circ} \mathrm{C}$ mittlerer Tagesmitteltemperatur am größten ist. • Abb. 3 zeigt die Verteilung der relativen Häufigkeiten für Sterbeexzesse in Abhängigkeit von der mittleren Tagesmitteltemperatur auf Basis der unbereinigten Erwartungswertschätzung für die Jahre 2005 bis 2012. Wie sich zeigt, steigt die relative Häufigkeit für einen Sterbeexzess $a b$ einer mittleren Tagesmitteltemperatur von $23,5^{\circ} \mathrm{C}$ stark an. Als Expositionsschwelle sowohl für die Bereinigung der Erwartungswerte als auch als Expositionsschwelle für das zu untersuchende Jahr wurde daher eine Temperatur von $23^{\circ} \mathrm{C}$ als die Mitte des davorliegenden Temperaturintervalls $\left(22,5-23,4^{\circ} \mathrm{C}\right)$ gewählt.

Für eine Pilotstudie zu einer kleinräumigeren Betrachtung des hitzebedingten Sterbegeschehens wurde auf

Bundesgesundheitsbl 2019 -62:580-588 https://doi.org/10.1007/s00103-019-02941-x ○ Der/die Autor(en) 2019

\section{H. Siebert $\cdot$ H. Uphoff $\cdot$ H. A. Grewe}

\section{Monitoring hitzebedingter Sterblichkeit in Hessen}

\section{Zusammenfassung}

Hintergrund. Der kontinuierlichen Beobachtung des Sterbegeschehens wird in aktuellen Empfehlungen für die Erstellung von Hitzeaktionsplänen eine hohe Bedeutung beigemessen. Sie dient der Problemerfassung und Maßnahmenevaluation. International existierende Monitoringsysteme unterscheiden sich in Datenauswahl und Auswertungsverfahren. In Deutschland dagegen sind etablierte Verfahren rar. Fragestellung. Wie lässt sich auf Landesebene ein praktikables Monitoring hitzeassoziierter Sterblichkeit mit Routinedaten etablieren? Material und Methoden. Basierend auf hessischen Sterbedaten wurden die Sommermonate 2000-2018 mit Wetterparametern in Beziehung gesetzt und Ansätze für räumliche Betrachtungen, Schwellenwertfestlegung und Bereinigungsverfahren exploriert.

Ergebnisse und Diskussion. Für das gesamte Landesgebiet in Hessen erwies sich die über alle Stationen gemittelte Tagesmitteltemperatur als geeigneter Temperaturparameter.

Die Schätzung der Basislinie erfolgte mittels gleitenden Mittels aus 25 Tageswerten auf Basis fünfähriger Referenzperioden, die Bereinigung der Erwartungswerte um Hitzeexzesse durch Ersetzung mit Beobachtungswerten aus drei Vorjahren. Als Exzesskriterium wurde das Zweifache der Standardabweichung der Erwartungswerte in Kombination mit einer Temperaturschwelle definiert, die aus der relativen Häufigkeit der Exzesssterbefälle je Temperaturintervall von $1{ }^{\circ} \mathrm{C}$ empirisch ermittelt wurde. Zwischen 2005 und 2018 konnten mit diesem Verfahren an 49 Tagen mit Tagesmitteltemperaturen $>23,0^{\circ} \mathrm{C}$ insgesamt 889 Exzesssterbefälle geschätzt werden.

Fazit. Eine zeitnahe Datenverfügbarkeit während der Sommermonate vorausgesetzt lässt sich mit diesem Verfahren ohne großen Aufwand eine kontinuierliche Beobachtung des Sterbegeschehens während der Sommermonate etablieren.

Schlüsselwörter

Routinedaten · Sterbeexzess · Klimawandel . Gesundheit · Temperatur

\section{Monitoring heat-related mortality in Hesse}

\section{Abstract}

Background. Continuous monitoring of the mortality phenomenon is given high priority in the current recommendations for the preparation of heat action plans in Germany with respect to problem detection and evaluation of interventions. International monitoring systems are heterogeneous concerning the procedures used. In Germany, such monitoring systems are rarely established. Objectives. Under what circumstances can a mortality monitoring system be operated on a regional basis using routine data? Materials and methods. Summer mortality data from Hesse from 2000 to 2018 and their associations with climate variables were analyzed. Different approaches regarding spatial analyses, definition of excess criteria, and adjusting procedures were explored. Results. In Hesse, daily mean temperatures averaged over all operating weather stations proved appropriate as a climate parameter. The expected daily number of deaths was estimated by a moving average based on 25 daily mortality datasets from reference periods of five years adjusted for mortality peaks using data from three previous years. Mortality excess was defined as twice the value of the standard deviation of the expected values including an empirically determined temperature threshold. This threshold was derived from analyzing relative frequencies of observed excess number of deaths per $1^{\circ} \mathrm{C}$ temperature interval. Based on this approach, 49 mortality excesses with a total of 889 excess deaths were estimated in Hesse during days with a daily mean temperature of more than $23.0^{\circ} \mathrm{C}$ during summer from 2005 to 2018.

Conclusions. The system described in this article turned out to be practicable for systematically monitoring mortality during summer. Timely availability of mortality and climate data is crucial.

Keywords

Routine data $\cdot$ Mortality excess $\cdot$ Climate change $\cdot$ Health $\cdot$ Temperature 


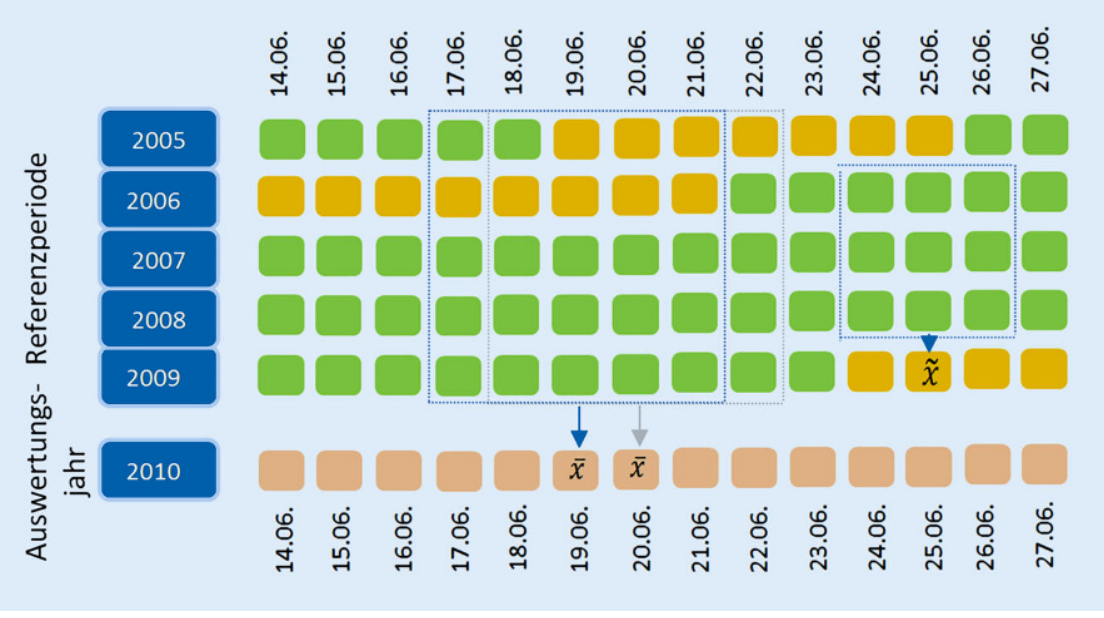

Abb. 2 \& Schema der Erwartungswertschätzung sowie des Bereinigungsverfahrens, Beispiel für das Auswertungsjahr 2010. Grüne Flächen repräsentieren Tage mit unbereinigten, beobachteten Fallzahlen während der Referenzperiode, gelbe Flächen repräsentieren Tage mit bereinigten Fallzahlen während der Referenzperiode, beigefarbene Flächen repräsentieren die Erwartungswerte für das Auswertungsjahr, $\bar{x}$ arithmetisches Mittel, $\widetilde{x}$ Median

den Ansatz der Testreferenzjahre (TRY) zurückgegriffen, die jeweils Zonen mit typischen klimatischen Charakteristika in Deutschland repräsentieren [19]. In Hessen dominieren zwei TRY-Regionen: die Region 7, die als Zone gemäßigten, in Höhenlagen kühlen Klimas mit mäBiger Durchlüftung den nördlichen Teil Hessens umfasst, und die Region 12, die durch ein im Winter gemäßigtes und im Sommer ausgeprägt warmes Klima vor allem den südlichen Teil Hessens repräsentiert [19]. Für die Regionalisierung wurden Sterbefälle derjenigen Landkreise berücksichtigt, die sich einer der beiden TRY-Regionen zuordnen ließen. Beide betrachteten Regionen umfassen zusammengenommen einen Anteil von rund $43 \%$ der Bevölkerung Hessens [20], die Region Nord etwa 1,2 Mio., die Region Süd etwa 1,5 Mio. Einwohner. Auf diese beiden Regionen wurde das entwickelte Auswertungsverfahren unter Anpassung des Temperaturkriteriums auf Basis für die jeweilige Region repräsentativer Wetterstationen angewendet.

Nach Abschluss der Entwicklungsphase wurden zur Überprüfung der Praktikabilität im Routinebetrieb anschließend die Sterbedaten des Zeitraumes 2013 bis August 2018 nach dem entwickelten Algorithmus ausgewertet.

Gemeinsam mit der Entwicklungsphase bildete damit eine Zeitreihe hessischer Sterbedaten aus den Jahren 2000 bis 2018 (bis einschließlich August) die Datenbasis für das hier vorgestellte Verfahren. Um im gesamten Datensatz eine Konsistenz der Fälle bezüglich Wohnort und Sterbeort herzustellen, wurden Fälle von in Hessen wohnhaften und nichtwohnhaften verstorbenen Personen einbezogen.

Die statistischen Analysen wurden mit R ([21]; v. 3.0.3.) und Microsoft Excel vorgenommen. Um die Anwendbarkeit zu erhöhen, wurde der Auswertungsalgorithmus in eine standardisierte und leicht $\mathrm{zu}$ bedienende Eingabemaske in Excel implementiert. Für den Endanwender steht damit eine aufwandsarme Möglichkeit jenseits der Verfügbarkeit statistischer Programmpakete zur Verfügung.

\section{Ergebnisse}

Das entwickelte Auswertungsverfahren erlaubt die Entdeckung von Tagen, an denen die Zahl der Sterbefälle über das noch als zufällig zu bezeichnende Maß hinausgeht und dieser Anstieg mit einer hohen Wahrscheinlichkeit durch hohe Außentemperaturen verursacht wurde. Das ermittelte Exzesskriterium für Hessen lautet

$$
b>(e+2 s d) \text { und } T(m)>23^{\circ} \mathrm{C}
$$

wobei $b$ die beobachteten und $e$ die erwarteten Sterbefälle, $s d$ die Standardabwei- chung der erwarteten Fälle und $T(m)$ die mittlere Tagesmitteltemperatur bezeichnet. Gezählt werden folglich diejenigen Sterbefälle, die oberhalb der statistischen Exzessgrenze an Tagen mit einer mittleren Tagesmitteltemperatur von mehr als $23^{\circ} \mathrm{C}$ aufgetreten sind. Die regionalisierten Schwellenwerte, die auf die gleiche wie für das gesamte Bundesland Hessen beschriebene Weise empirisch abgeleitet wurden, ergaben für die Regionen Nord und Süd Tagesmitteltemperaturen von $22,0^{\circ} \mathrm{C}$ bzw. $23,5^{\circ} \mathrm{C}$.

Die zunächst für den Zeitraum von 2000 bis 2012 und dann bis zum Jahr 2018 (bis einschließlich August) ausgeweitete Zeitreihe umfasste 1.101.450 Sterbefälle, davon ereigneten sich 265.233 (24,1\%) während der Sommermonate zwischen Juni und August. Im Jahresgang zeigt das Sterbegeschehen ein charakteristisches Muster mit einer hohen Fallzahl während der Wintermonate und einem insbesondere durch Influenzaaktivität zu erklärenden Gipfel im Februar, an den sich ein ausgeprägtes Tal der Fallzahlen während der Sommermonate anschließt, sowie einen langsamen Anstieg während der Herbst- und frühen Wintermonate (• Abb. 4). Auffällig sind die Jahre 2003 (im August) und 2015 (Anfang Juli), in denen während Hitzeperioden nahezu eine Verdoppelung der üblichen Fallzahlen zu beobachten war.

Die zeitlich enge Beziehung zwischen Temperaturverlauf und Sterbegeschehen wird exemplarisch anhand der Jahre 2003 und 2015 in - Abb. 5 deutlich.

Für das gesamte Bundesland zeigten sich im Auswertungszeitraum (2005 bis August 2018) während der 1288 Sommertage 889 wahrscheinlich durch hohe Außentemperaturen verursachte zusätzliche Sterbefälle an 49 Exzesstagen (18,1 Exzesssterbefälle/Exzesstag). Es treten die Jahre 2006, 2010, 2012, 2015 und 2018 hervor, die $799(89,9 \%)$ aller Exzesssterbefälle und 37 (75,5\%) aller Exzesstage auf sich vereinen (• Abb. 6). In der regionalen Betrachtung sind es für die Region Hessen Nord insbesondere die Jahre 2008, 2010 und 2012, auf die zusammen 28 (64\%) aller dort beobachteten Exzesssterbefälle entfallen. Dagegen sind es für Hessen Süd die Jahre 2010 sowie 2012, während denen 57 zu- 


\section{Leitthema}

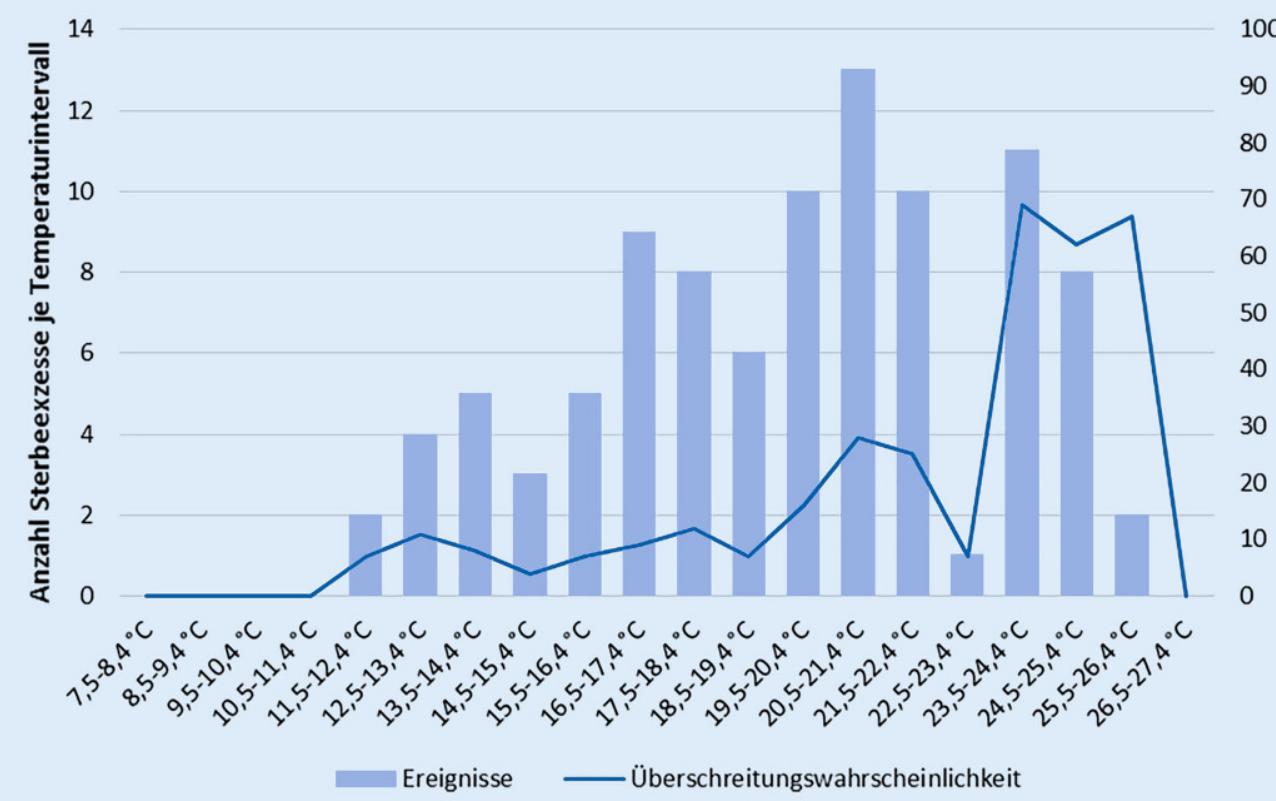

Abb. $3 \triangleleft$ Absolute und relative Häufigkeiten für das Auftreten mindestens eines Exzesssterbefalls (Ereignis) je Temperaturintervall (2005 bis 2012). Temperatur ist das Flächenmittel der mittleren Tagesmitteltemperatur $\left({ }^{\circ} \mathrm{C}\right)$

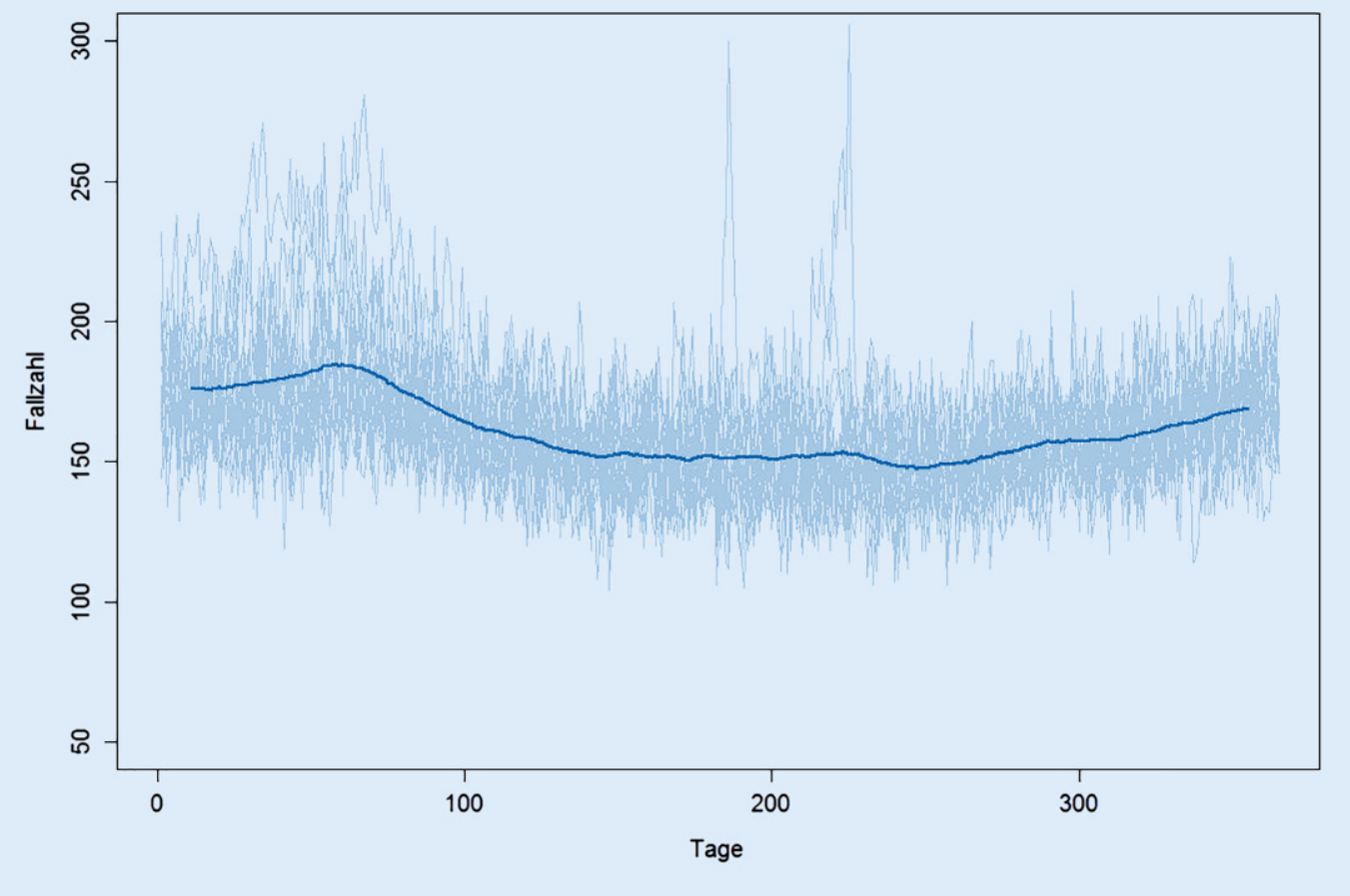

Abb. 4 Sterbefälle im Zeitraum 2000 bis 2018 in Hessen im Jahresgang (Januar bis Dezember*, **). Hellblaue Linien: Sterbefälle jeweils vom 01.01 . (Tag 0) bis 31.12. (Tag 365) der Jahre 2000 bis 2018, dunkelblaue Linie: durchschnittliche Anzahl aller Sterbefälle (Glättung mittels gleitenden Mittels über 21 Tageswerte), *für 2018 nur bis 31.08.; ** Sterbefallpeak 2015 (um Tag 186), Sterbefallpeak 2003 (um Tag 225)

sätzliche wahrscheinlich durch Hitze verursachte Sterbefälle ( $85 \%)$ geschätzt wurden.

- Tab. 1 dokumentiert die Ergebnisse für das Bundesland sowie der regionalisierten Auswertung. Für Hessen gesamt wurde die Expositionsschwelle von $23^{\circ} \mathrm{C}$ mittlerer Tagesmitteltemperatur im untersuchten Zeitraum an 91 Tagen überschritten, von denen 49 Tage (54\%) einen
Sterbeexzess zeigten. Im Durchschnittereigneten sich 18,1 Exzesssterbefälle je Exzesstag. In der nach regionalen Kriterien differenzierten Pilotstudie über den Zeitraum 2005 bis 2012 zeigt Hessen Nord zehn Exzesstage mit 44 Exzessfällen (4,4 Exzesssterbefälle/Exzesstag), basierend auf 736 Sommertagen. In der Region Hessen Süd wurden für denselben Zeitraum 15 Exzesstage mit 67 Exzess- fällen beobachtet (4,5 Exzesssterbefälle/ Exzesstag). Obschon diese Befunde auf eine unterschiedliche Betroffenheit im Regionalvergleich hinweisen, muss eine routinehafte Implementierung der regionalen Differenzierung Gegenstand einer längerfristigen Evaluation sein und kann zum derzeitigen Stand noch nicht abgesehen werden. 


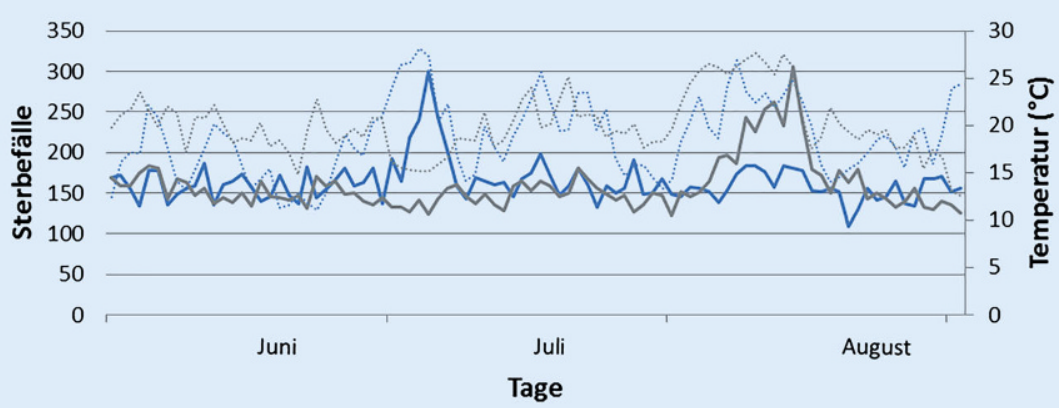

Abb. 5 ॥ Zeitliche Beziehung derbeobachteten Sterbefälle und der mittleren Tagesmitteltemperatur, Sommermonate 2003 und 2015. Graue durchgezogene Linie: beobachtete Sterbefälle vom 01.06.2003 bis 31.08.2003, graue unterbrochene Linie: mittlere Tagesmitteltemperatur $\left({ }^{\circ} \mathrm{C}\right)$ vom 01.06 .2003 bis 31.08.2003; blaue durchgezogene Linie: beobachtete Sterbefälle vom 01.06 .2015 bis 31.08.2015, blaue unterbrochene Linie: mittlere Tagesmitteltemperatur $\left({ }^{\circ} \mathrm{C}\right)$ vom 01.06 .2015 bis 31.08 .2015

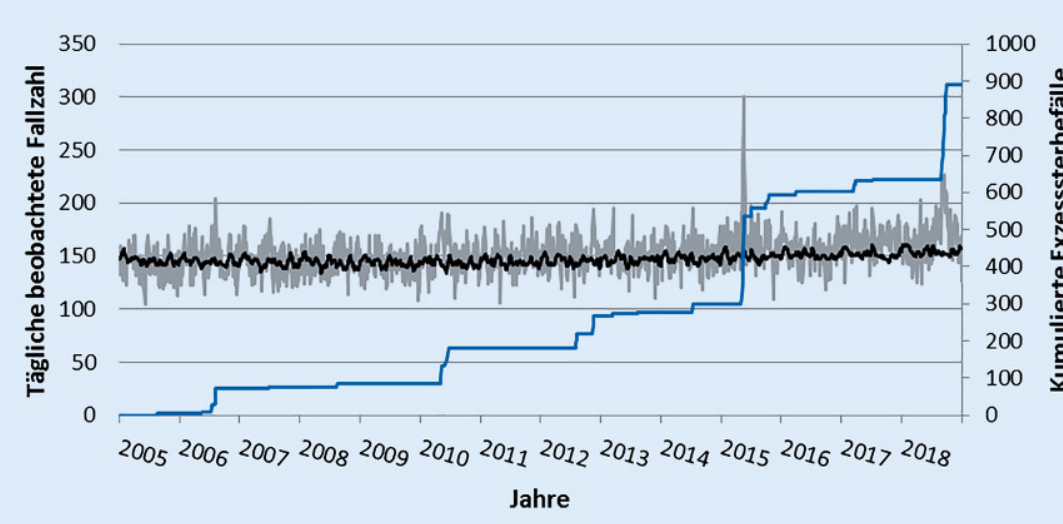

Abb. 6 A Beobachtete und erwartete Sterbefälle sowie kumulierte Exzesssterbefälle für Hessen gesamt, Sommermonate 2005 bis 2018 . Blassgraue Linie: beobachtete Sterbefälle, schwarze Linie: erwartete Sterbefälle, blaue Linie: kumulierte Exzesssterbefälle

Das Verfahren hat sich im Rahmen der Pilotphase als einfach umsetzbar und aufwandsarm erwiesen. Die Datensparsamkeit wird durch die Definition eines Minimaldatensatzes gewährleistet, der von der zukünftig mit der Schätzung der Exzesssterbefälle beauftragten Auswertungsstelle verwendet werden kann [22].

\section{Diskussion}

Ausgehend von einer Zusammenschau international existierender Monitoringsysteme wurden Rahmenbedingungen abgeleitet, unter denen ein solches System im Routinebetrieb umgesetzt werden kann.

Trotz der Heterogenität etablierter Verfahren ist es Konsens, dass ein nutzbringend operierendes Monitoringsys- tem ein den klimatischen Bedingungen, soziodemografischen Strukturen und dem jeweils geltenden Normensystem angepasstes Vorgehen umfassen soll. Eine kritiklose Übernahme der Verfahrensweisen anderer Systeme, etwa des Euro-MOMO-Algorithmus, erschien damit weder sinnvoll noch zielführend. Der MOMO-Algorithmus schätzt Exzesssterbefälle aus wöchentlich zusammengefassten Sterbedaten, die Referenzperioden werden aus relativ kurzen Anteilen aus den meteorologischen Frühlings- und Herbstmonaten entnommen [18]. Unter Berücksichtigung meteorologischer und medizinisch-klinischer Expertise wurde hier dagegen einem anderen Ansatz gefolgt, der vorsieht, die Erwartungswerte anhand des Sterbegeschehens während der Sommermonate zu schätzen. Dadurch ist gewährleis- tet, dass das Verfahren das tatsächliche Sterbegeschehen in den Sommermonaten berücksichtigt. Im Ergebnis schätzt dieses Verfahren eine Basislinie auf der Grundlage empirisch beobachteter Fallzahlen und nicht auf der Grundlage statistischer Modellierung aus Ausschnitten von Daten aus Frühlings- und Herbstmonaten. Das beschriebene Bereinigungsverfahren eliminiert dabei die verzerrenden Einflüsse zurückliegender Sterblichkeitsexzesse. Dadurch grenzt sich das hessische Monitoringsystem auch von jenem in Baden-Württemberg ab. Letzteres schätzt Erwartungswerte auf Basis einer Auswahl einzelner Monate aus einer langen Zeitperiode unter der Annahme, dass diese das von Hitze unbeeinflusste Sterbegeschehen repräsentieren. Anders als in Hessen beinhaltet das baden-württembergische System keine statistische Exzessgrenze, sondern weist die Summe der während der Sommermonate über der Basislinie liegenden Sterbefälle als hitzebedingten Exzess aus. Darüber hinaus ist darin auch keine Temperaturschwelle integriert, $a b$ deren Überschreiten von einem hitzebedingten Exzess gesprochen wird. In Relation zu diesem Vorgehen erscheinen die hessischen Befunde aufgrund des restriktiveren Exzesskriteriums als konservativer, zugleich vor dem Hintergrund der Temperatur als Expositionsvariable als intuitiver.

Das entwickelte Monitoringsystem weist Limitationen auf. Zunächst erscheint die Annahme eines hinsichtlich Gesamtfallzahl und Jahresgang relativ konstanten Sterbegeschehens, obgleich für fünfjährige Zeitintervalle plausibel, als kritisch, denn über längere Beobachtungszeiträume zeigt sich ein Trend zunehmender Sterbefallzahlen als natürliche Folge demografischer Alterung [23]. Damit besitzt der Algorithmus, der die erwarteten Fallzahlen aus historischen Referenzperioden tendenziell unterschätzt, Verzerrungspotenzial in Richtung einer systematischen Überschätzung der Exzesssterbefälle. Der Einfluss auf die Ergebnisse ist nach Auffassung der Autoren jedoch zu vernachlässigen, da eine lediglich 5 Jahre umfassende, prospektiv mitlaufende Referenzperiode zur Erwartungswert- 


\begin{tabular}{|l|l|l|l|l|l|}
\hline Tab. 1 Exzesstage und Exzesssterbefälle in Hessen gesamt und nach Region \\
\hline $\begin{array}{l}\text { Region/Auswertungs- } \\
\text { zeitraum }\end{array}$ & $\begin{array}{l}\text { Temperatur- } \\
\text { schwelle } \\
{\left[{ }^{\circ} \mathbf{C}\right]}\end{array}$ & $\begin{array}{l}\text { Tage ober- } \\
\text { halb Tem- } \\
\text { peratur- } \\
\text { schwelle }\end{array}$ & $\begin{array}{l}\text { Davon } \\
\text { Exzesstage } \\
\text { (Anteil \%) }\end{array}$ & $\begin{array}{l}\text { Exzess- } \\
\text { fälle }\end{array}$ & $\begin{array}{l}\text { Exzess- } \\
\text { fälle je } \\
\text { Exzesstag }\end{array}$ \\
\hline $\begin{array}{l}\text { Hessen gesamt } \\
(2005-2018)\end{array}$ & $>23$ & 91 & $49(54)$ & 889 & 18,1 \\
\hline $\begin{array}{l}\text { Hessen Nord } \\
(2005-2012)\end{array}$ & $>22$ & 42 & $10(24)$ & 44 & 4,4 \\
\hline $\begin{array}{l}\text { Hessen Süd } \\
(2005-2012)\end{array}$ & $>23,5$ & 69 & $15(22)$ & 67 & 4,5 \\
\hline
\end{tabular}

schätzung herangezogen wird, durch die eine „lernende“ Basislinie mit sukzessive sich vergrößernden Erwartungswerten geschätzt wird. Aus methodischer Sicht wäre eine nach demografischen Kriterien wie etwa dem Alter adjustierte Auswertung wünschenswert, setzt bei tagesgenauen Sterbedaten allerdings die Kenntnis der Populationsgröße in gleicher zeitlicher Auflösung voraus. Die Verwendung des Mittelwertes der Populationsgröße würde eine Vergröberung bedeuten, in deren Folge Fehleinschätzungen der Anzahl der Exzesssterbefälle im Kontext insgesamt recht kleiner Fallzahlen wahrscheinlich wären. Darüber hinaus ist zu berücksichtigen, dass der hier dargestellte Algorithmus die Anzahl an Exzesssterbefällen und Exzesstagen aufgrund der Kombination aus statistischer Exzessschwelle und einer Temperaturschwelle eher konservativ schätzt. $\mathrm{Da}$ ein sinnvolles und valides externes Kriterium derzeit nicht existiert, bleibt unklar, ob diese konservative Schätzung tatsächlich in relevantem Umfang zu einer Unterschätzung der hitzebedingten Todesfälle führt. Hinzu kommt das Problem eingeschränkter Attribuierbarkeit, das, zumal in Unkenntnis der Todesursachen, eine zweifelsfreie Zuschreibung eines durch den Algorithmus signalisierten Exzesssterbefalls als Fall, der tatsächlich durch Hitzeeinfluss hervorgerufen wurde, unmöglich macht. Eine solchermaßen limitierte Attribuierbarkeit ist, insbesondere aufgrund der Komplexität der Zusammenhänge, daher ein lediglich anteilig mittels Plausibilitätsannahmen auflösbares Problem. Eine Bewertung der Präzision der Schätzungen für die erwarteten Sterbefälle erscheint problematisch, da bei Angabe eines Konfidenzbereiches relevante weitere $\mathrm{zu}$ zusätzlicher Unsicherheit führende Faktoren (z. B. urlaubsbedingte Fluktuationen, Unfallgeschehen durch Krafträder durch höhere Nutzungsfrequenz im Sommer) unberücksichtigt blieben und eine Sicherheit vorgetäuscht würde, die in Wirklichkeit nicht gegeben ist.

Gemäß der Prämisse, ein für alle Akteure gut nachvollziehbares und akzeptables Verfahren zu entwickeln, basiert der Algorithmus auf einem recht einfachen statistisch-methodischen Ansatz. Demgegenüber sind in anderen Monitoringsystemen anteilig komplexere statistische Auswertungsroutinen, etwa regressionsbasierte Schätzungen der Erwartungswerte, implementiert. Von der Überlegenheit eines komplexen im Vergleich zu einem weniger komplexen Ansatzes kann jedoch nicht pauschal ausgegangen werden [24]. Der Algorithmus soll aufgrund seiner Einfachheit und guten Interpretierbarkeit insbesondere den Akteuren des öffentlichen Gesundheitsdienstes entgegenkommen.

Das hier vorgestellte Verfahren repräsentiert das vorläufige Ende eines Entwicklungsprozesses, in dem statistische, epidemiologische, medizinische sowie meteorologische Aspekte berücksichtigt wurden. Es ist an die Verfahrensweisen internationaler Systeme angelehnt und berücksichtigt zugleich die spezifischen hessischen Gegebenheiten, die etwa aus der Heterogenität der in Hessen gegebenen klimatischen Bedingungen resultieren. Als Ergebnis eines Prozesses des Suchens und Erprobens verschiedener Ansätze konnten wesentliche Entscheidungen zur Gestaltung des Algorithmus aus den Auswertungen der empirischen Daten heraus begründet werden. Andererseits ist ein Mortalitätsmonitoring nicht ohne epidemiologische, medizinische sowie demografische Plausibilitätsannahmen und Setzungen möglich.

Die Robustheit und Praktikabilität des entwickelten Algorithmus soll im Routinebetrieb getestet und fortlaufend evaluiert werden. So ist z.B. zu prüfen, ob die Beschränkung des durch das Monitoringsystem beobachteten Zeitraums (Juni bis August) ggf. zu kurz ist, um das hitzebedingte Sterbegeschehen vollständig erfassen zu können. Das hier vorgestellte System ist demzufolge zukünftig dahin gehend zu überprüfen, ob eine Ausweitung des Zeitraums um die Monate Mai und September von Nutzen sein kann. Darüber hinaus sollte die Frage, inwiefern eine wie im Rahmen der Pilotstudie vorgenommene Regionalisierung der Exzessschätzung sinnvoll ist, Gegenstand der Evaluation sein. Durchaus könnte der aus den Befunden entnommene vorsichtige Hinweis unterschiedlich starker Betroffenheit in den beiden Regionen ein Argument für eine regionale Betrachtung sein. Hier eröffnet sich zukünftiger Forschungsbedarf, der neben einer langfristigen, physiologischen Adaptionsfähigkeit auch regionale Unterschiede individuellen Verhaltens wie etwa Verschattungspraktiken zur Kühlung von Innenräumen oder die Konformität mit Empfehlungen zur Verhaltensanpassung (ausreichende Flüssigkeitszufuhr, Meidung körperlicher Aktivität im Freien) adressiert. Darüber hinaus erscheint auch von besonderer Relevanz, das Hitzemanagement von Institutionen wie Kindertagesstätten, Schulen oder Altenpflegeeinrichtungen unter dem Aspekt regionaler Differenzierung stärker in den Blick zu nehmen.

Die Auswerteprozeduren sind so angelegt, dass tagesgenaue Daten verarbeitet und zeitnahe Analysen des Sterbegeschehens erfolgen können. Tagesgenaue Sterbedaten können unter den in Hessen derzeit geltenden Datenflusswegen jedoch nicht in „Echtzeit" bereitgestellt werden. Gemäß Personenstandsgesetz (PStG) ist ein Todesfall spätestens am dritten auf den Todestag folgenden Werktag an das zuständige Standesamt zu melden, wo er nach Prüfung und Beurkundung in das Sterberegister aufgenommen wird. Die Todesfälle 
werden anschießend an die zentrale Dateneingangsstelle (Bayern) und an das zuständige Gesundheitsamt bzw. weitere Behörden übermittelt [25]. Zur Bearbeitung werden die Daten von Bayern aus an die zentrale Daten-Verarbeitungsstelle in Thüringen weitergeleitet und durch die Statistischen Landesämter ausgewertet. Durch primäre Aufnahme und Prüfung laufen die Daten mit einer gewissen Verzögerung auf, wobei aber bereits in der Folgewoche über $90 \%$ aller Sterbefälle erfasst sind. Für ein zeitnahes Monitoring sollten die bestehenden Datenflussstrukturen so angepasst werden, dass eine Übermittlung aktueller Daten mindestens auf wöchentlicher Ebene erfolgen kann. Darüber hinaus bedarf es einer Anbindung an eine Institution, die neben der infrastrukturellen und EDVtechnischen Umgebung auch die Verfügbarkeit epidemiologischer Expertise für die Interpretation der Auswertungsergebnisse sicherstellen kann.

Während in den meisten existierenden Verfahren Konzepte von Hitzeperiode bzw. Hitzewelle insbesondere über Kriterien wie Ausmaß und Dauer der Hitzeexposition entwickelt wurden (vgl. etwa $[10,13,26])$, wurde hier auf die Einführung einer Mindestdauer des Vorliegens hoher Außentemperaturen zur Definition eines Hitzeereignisses verzichtet, zumal hohe Temperaturen und ansteigende Sterbefallzahlen zeitlich eng zusammenhängen. Unklar bleiben etwa auch der Einfluss kühlerer Tage, die unmittelbar auf heiße Tage folgen, sowie der Einfluss von Hitzeereignissen mit langer Dauer. Aus diesem Grund wurde der Ansatz verfolgt, jeden Tag mit einer hohen Tagesmitteltemperatur als singuläres, potenziell bedeutsames Hitzeereignis aufzufassen.

\section{Fazit}

Monitoringsysteme zur Dauerbeobachtung hitzebedingter Sterblichkeit sind international zunehmend etablierte Instrumente zur Gesundheitsfolgenabschätzung im Kontext von Strategien zur Anpassung an die Folgen des Klimawandels. Als Teil von Hitzeaktionsplänen tragen sie dazu bei, die Bevölkerung vor Gesundheitsgefährdungen durch hohe
Außentemperaturen zu schützen. Für Hessen wurde mit dem vorgestellten Algorithmus ein Vorschlag vorgelegt, der die Angemessenheit der statistischen Verfahren, die Praktikabilität in der Umsetzung sowie die Verständlichkeit auch für die Laienöffentlichkeit gewährleistet. Gleichwohl gilt es zu evaluieren, ob sich die nach der Überführung in den Routinebetrieb etablierten Strukturen bewähren konnten und an welchen Stellen das Verfahren modifiziert werden sollte. Hierfür sind Indikatoren $\mathrm{zu}$ entwickeln, die aus einer hessischen Gesamtstrategie zur gesundheitsbezogenen Anpassung an den Klimawandel abgeleitet werden können.

\section{Korrespondenzadresse}

\section{Hendrik Siebert}

Public Health Zentrum, Hochschule Fulda Leipziger Straße 123, 36037 Fulda, Deutschland hendrik.siebert@pg.hs-fulda.de

Danksagung. Wir danken dem Hessischen Landesamt für Naturschutz, Umwelt und Geologie (HLNUG) für die Finanzierung des Projektes HEAT II sowie Susanne Schroth für die umsichtige Projektbetreuung. Wir danken Herrn Werner Hofmann (Hochschule Fulda) für die fortgesetzte Einbringung seiner statistischen Expertise.

\section{Einhaltung ethischer Richtlinien}

Interessenkonflikt. H. Siebert, H. Uphoff und H.A. Grewe geben an, dass kein Interessenkonflikt besteht.

Für diesen Beitrag wurden von den Autoren keine Studien an Menschen oder Tieren durchgeführt. Für die aufgeführten Studien gelten die jeweils dort angegebenen ethischen Richtlinien.

Open Access. Dieser Artikel wird unter der Creative Commons Namensnennung 4.0 International Lizenz (http://creativecommons.org/licenses/by/4.0/deed. de) veröffentlicht, welche die Nutzung, Vervielfältigung, Bearbeitung, Verbreitung und Wiedergabe in jeglichem Medium und Format erlaubt, sofern Sie den/die ursprünglichen Autor(en) und die Quelle ordnungsgemäß nennen, einen Link zur Creative Commons Lizenz beifügen und angeben, ob Änderungen vorgenommen wurden.

\section{Literatur}

1. Robine JM, Cheung SL, Le Roy S et al (2008) Death toll exceeded 70,000 in Europe during the summer of 2003. CRBiol 331:171-178

2. Li M, Gu S, Bi P, Yang J, Liu Q (2015) Heat waves and morbidity: current knowledge and further direction - a comprehensive literature review. Int JEnviron Res Public Health 12:5256-5283

3. Bouchama A, Dehbi M, Mohamed G, Matthies F, Shoukri M, Menne B (2007) Prognostic factors in heat wave related deaths: a meta-analysis. Arch Intern Med 167:2170-2176

4. Bunker A, Wildenhain J, Vandenbergh A et al (2016) Effects of Air Temperature on ClimateSensitive Mortality and Morbidity Outcomes in the Elderly; a Systematic Review and Meta-analysis of Epidemiological Evidence. EBioMedicine 6:258-268

5. Robert Koch-Institut, Umweltbundesamt (2013) Klimawandel und Gesundheit. Allgemeiner Rahmen zu Handlungsempfehlungen für Behörden und weitere Akteure in Deutschland. Berlin, Robert Koch-Institut, Epidemiologie und Gesundheitsberichterstattung

6. Grewe H, Blättner B (2011) Hitzeaktionspläne in Europa. Strategien zur Bekämpfung gesundheitlicher Folgen von Extremwetterereignissen. Präv Gesundheitsf 2011:158-163

7. World Health Organisation (2008) Heat Health Action Plans. Guidance

8. Nogueira PJ, Machado A, Gil AP et al (2010) European monitoring of excess mortality for public health action, euroMOMO. Work Package 5 Report. Concept: Core attributes and requirements. Lissabon: ReportEuro-Momo-Initiative

9. Mazick A (2007) Monitoring excess mortality for public health action: potential for a future European network. Euro Surveill. 12(1):pii=3107

10. Cox B, Wuillaume F, Van Oyen H, Maes S (2010) Monitoring of all-cause mortality in Belgium (BeMOMO): a new and automated system for the early detection and quantification of the mortality impact of public health events. Int J Public Health 55:251-259

11. Laaidi K, Ung A, Wagner V, Beaudeau P, Pascal M (2013) The French Heat and Health Watch Warning System: principles, fundamentals and assessment

12. Pascal M, Laaidi K, Ledrans $M$ et al (2006) France's heat health watch warning system. Int J Biometeorol 50:144-153

13. Nogueira PJ, Machado A, Rodrigues E et al (2010) The new automated daily mortality surveillance system in Portugal. Euro Surveill 15:pii:19529

14. Be-Momo (2017) Belgian Mortality Monitoring. Scientific Institute of Public Health (WIV-ISP), Brüssel, Belgien. https://epistat.wiv-isp.be/momo/. Zugegriffen: 25 . Oktober 2017

15. Green HK, Andrews NJ, Bickler G, Pebody RG (2012) Rapid estimation of excess mortality: nowcasting during the heatwave alert in England and Wales in June 2011. J Epidemiol Community Health 66:866-868

16. Brachat-Schwarz W, Winkelmann U (2017) Führt der Klimawandel zu einem Anstieg der "Hitzetoten"? Zur Abschätzung der Sterbefälle aufgrund hoher Temperaturen in. Württemberg, Bd. 2017. Württemberg. Statistisches Monatsheft Baden, Baden

17. (2018) https://www.statistik-bw.de/Presse/ Pressemitteilungen/2018168 (Erstellt: 10.2018)

18. Gergonne B, Mazick A, O'donnell J et al (o.J.) European monitoring of excess mortality for public health action, euroMOMO. Work Package 7 Report. A European algorithm for a common monitoring of mortality across Europe. Kopenhagen

19. Bundesamt Für Bauwesen Und Raumordnung (Bbr) (2014) Testreferenzjahre von Deutschland für mittlere, extreme und zukünftige Witterungsverhältnisse 


\section{Leitthema}

20. Hessisches Statistisches Landesamt (2018) Statistische Berichte. Die Bevölkerung der Hessischen Gemeinden am 31. Dezember 2017. Fortschreibungsergebnisse Basis Zensus 09. Mai 2011

21. RCore Team (2017) R: Alanguage and environment for statistical computing. R Foundation for Statistical Computing, Vienna, Austria. http:// www.R-project.org/. Zugegriffen 30. Oktober 2017

22. Hessisches Landesamt Für Naturschutz Umwelt Und Geologie (2017) Etablierung eines Surveillance-Systems für hitzebedingte Mortalität in Hessen-HEATII. Schlussbericht

23. Statistisches Bundesamt (2017) Statistisches Jahrbuch. Deutschland und Internationales.

24. Strat $Y$ (2005) Overview of temporal Surveillance. In: Lawsen A, Kleinman K (Hrsg) Spatial and Syndromic Surveillance for Public Health. Wiley, Chichester

25. Uphoff H, Geis S, Wirtz A, Hauri A (2011) Zeitnahe Erfassung und Übermittlung von Todesfällen in Hessen. Eine erste Einschätzung zur Influenza-A/H1N1v-Pandemie. Bundesgesundheitsbl Gesundheitsforsch Gesundheitsschutz 2011:867-874

26. Michelozzi P, De' Donato FK, Bargagli AM et al (2010) Surveillance of summer mortality and preparedness to reduce the health impact of heat waves in Italy. Int J Environ Res Public Health 7:2256-2273 